\title{
Many Independent Origins of trans Splicing of a Plant Mitochondrial Group II Intron
}

\author{
Yin-Long Qiu, ${ }^{1,2}$ Jeffrey D. Palmer ${ }^{1}$ \\ ${ }^{1}$ Department of Biology, Indiana University, Bloomington, IN 47405, USA \\ ${ }^{2}$ Department of Ecology and Evolutionary Biology, University of Michigan, Ann Arbor, MI 48109, USA
}

Re: J Mol Evol (2004) 59:80-89. Due to a printer's error, Fig. 3 of this article did not appear correctly in the printed issue. We present here the corrected Fig. 3 along with its caption.

Fig. 3. Phylogenetic distribution of trans splicing of nad1i728 in land plants. The phylogeny shown was generated as described in Materials and Methods. The species with trans-spliced introns are shown in gray boxes (except for Hedychium coronarium and Pontederia cordata, which may have secondarily derived cis-spliced introns and are indicated by asterisks, and Amborella trichopoda, whose intron status is even less clear and is therefore marked by a question mark). The lineages whose trans-spliced introns are broken between the 5'-end of the intron and mat $R$ are shown by thick gray lines, while those with breakage between mat $R$ and the 3'-end of the intron are shown by thick black lines. For taxa with a black dot, the splicing status of the intron was determined by sequencing (see text for citations in which the sequences were reported). For taxa in italics, the splicing status of the intron was inferred using Southern hybridizations with four single-enzyme digests and 12-cm electrophoretic separation (many of these taxa were also investigated in the first round of hybridizations using two other, single-enzyme digests and 6-cm electrophoretic separation). All other taxa (in plain text) have a cis-spliced intron according to the results of Southern hybridizations using two single-enzyme digests and $6 \mathrm{~cm}$ electrophoretic separation. NYM-Nymphaeales, AUS-Austro-baileyales, CHL-Chloranthaceae, WIN-Winteraceae, CYM-Cymdoceaceae, HYD-Hydrocharitaceae, NAJ-Najadaceae, EUM-Eumonocots, POL-Polygonales, CAR-Caryophyllales, ERO-Erodium, SAP-Sapindales, ROS-Rosales, JUG-Ju-glandaceae, SOL-Solanaceae. 

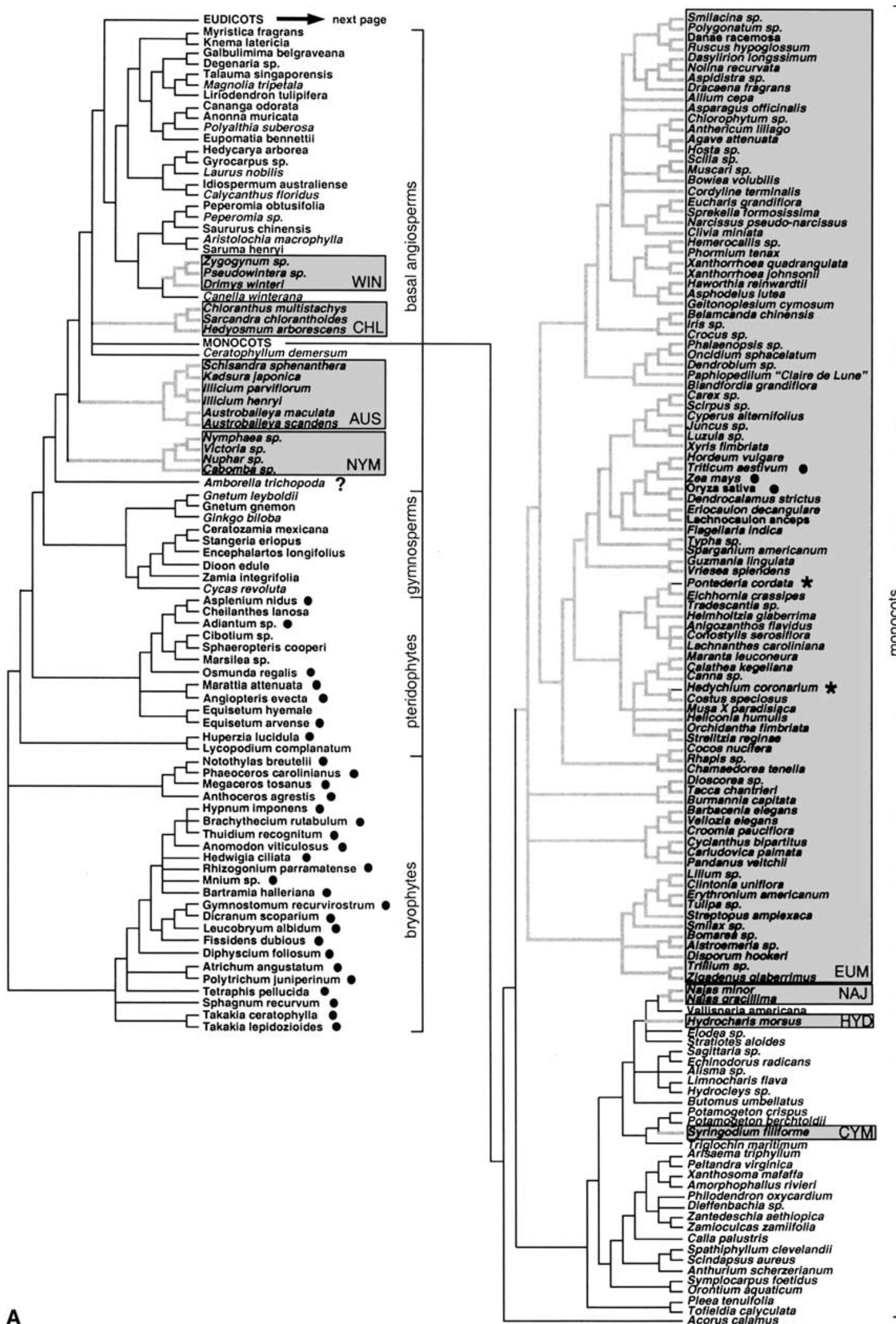
Castanea pumila

Chrysolepis sempervirens

Betula nigr

C Casuarina litore

- Myrica cerifera

-EL Jualans clinera

- Carya sp.

$-\leftarrow$ Cutfa acutangula

Cucumis sativus

- Datisca glomera

Coriarigmyrtiolit

Colths yunnanensis

Humulus lupulus

Morus alba

Ficus diversiffolla

Pllea fontana

Boehmeria nivea

Uimus pumila

limus thomsil

Hovenla dulcls

Rhamnus catharticus

Elaeagnus so.

Elaeagnus se.

Malus domestica

Glycine max

- Vicia faba

-Medicago lupulin

- Medicago sativ

Calliandra inaequilater

Cercis canadensis

Polygala lindheimer

Acalypha sp.

Euprocbia milli

C Croton sp.

Hevea brasillensis

Malpighia glabra

Salix sp.

Passifilora suberosa

Erythroxylum coca

Hypericum sp.

Erexyla madagascarensis

Oxalls so.

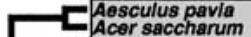

Bursera sp.

Rhus radican

Crirus himon

- Triphasla trifollo

- Ptelea trifollata

- Liltnerla floridane

- Quararibea aterolepis

Abutilon theophrasti

Cardamine sp.

- Arabidopsis thallana

Carica papaya

Vochysia sp.

Eugenia unitiora

Psidium gualava

Callistemon lanceolatus

Eucalyptus cinerea

Cenothera berteriana

enethera organensis

rodium coldum yoles

Erodium tritollum

Geranlum macrorrhizum

Staphylaa trifolit

Vitis sp.

Loropetalum chinense

Corylopsis gotoana

Distylium racemosum

Hamamelis mollis

Mytilaria laosensis

Cercidiphyllum

Liquidambar styraciflua

Saxifraga sarmentosa

Heuchera sp.

- Itea virginica

Ribes sp.

Myriophyllum sp.

CARYOPHYLUIDS

Leplonurus sylvestris

Trochodendron aralioides

Tetracentron sinense

Buxus sp.

Pachysandra procumbens

Sabia sp.

- Grevillea robusta

- Platanus occidental

Ranunculus sp.

Ranunculus sp.

Clematis sp.

Podophyllum peltatum

Mahonla beale

Clssampelos pareira

Cocculus trilobus

Akebla quinata

Euptelea poly

Dicentra sp.

Bocconia fructescens

B

Sanguinaria canadensis
Campsis grandillora

- Catalpa fargesel

- Scutellarla mociniana

Lamium sp.

Clerodendrum trichotomum

- Justicla americana

Sanchezla nobllis

Thunbergla erecta

- Pingulcula vulgaris

- Byblis linifolla

- Callitriche heterophylla

Hebe subalping

- Scrophularia nodosa

Asarina antirrminiflora

Verbascum sp.

Buddleja lindleyane

Drymonla serrulata

Olea europaea

L Syringa sp.

Forsythla sp.

Heliotroplum arborescens

L Phacella sp.

- Symphytum officinale

L Mertensia virginica

- Hoya sikkimensis

- Carissa sp.

- Catharanthus roseus

- Gentiana macrophylla

- Ixora sp.

- Coffea arablca

- Nicotiana tabacum

Petunia $X$ hybrida - SOL

Capsicum annuum

Ipomoea batatas

Eucommla ulmoldes

- Daucus carota

Hydrocotyle rotundifolia

- Hedera helix

- Schefflera actinophylla

- Pittosporum tobira

- Sambucus canadensis

Viburnum sp.

- Lonicera sp.

- Hellanthus annuus

- Lactuca sativa

- Calycera sympaganthera Goodenla ovate

Menyanthes trifoliate

- Trachelium caeruleum

Prismatocarpus fruiticosus

Platycodon grandiflora

- llex sp.

- Chimaphila sp.

- Pyrola rotundifol

Pyrola secund

Andromeda glaucophylla

Chamaedaphne calyculat

Sarracenia flava

Actinidia argutz

Cyrilla racemiflora

Clethra barbinervis

Diospyros virginiana

Symplocos panic

Camelila sinensis

Achras zapota

Fouquieria columnaris

- Ardisia sp.

Primula vulgaris

Impatiens pallida

Halesia carolina

- Deutzla gracilia

Hydrangea arborescens

Eucnido hirta

- Cornus foemina

- Alanglum platanifollum

Camptotheca acuminate

Davidia involucrata

Notocactus leninghaus

Pereskla grandifolls Portulaca oleracea Mollugo verticiliata Phytolacca american

Oscularia sp.

Bougalnvilles sp. Stegnosperma hallmifolia

- Beta vulgaris ?

Splnacla oleraces

Chenopodlum sp.

Colosla cristata

C Silene acaulls Simmendslac

Promo sp.

Fagopyrum esculentum

Polygonum sp.

Tamarix so.

Dionaea muscipula

Nepenthes $X$ kosobe 